\title{
Tradeoffs between attentional effects of spatial cues and abrupt onsets
}

\author{
JAMES F. JUOLA \\ University of Kansas, Lawrence, Kansas \\ and Institute for Perception Research/IPO, Eindhoven, The Netherlands \\ and \\ HIDEYA KOSHINO and C. BRUCE WARNER \\ University of Kansas, Lawrence, Kansas
}

\begin{abstract}
We determined the relative effectiveness and tradeoffs among central, peripheral, and abrupt onset cues in directing attention to a potential target character. Central cues were arrows located at the fixation point, whereas peripheral cues were arrows occurring about $3^{\circ}$ away from fixation, near the location of a potential target. These were contrasted with the abrupt onset of an ambiguous part of a character, which later was filled in to reveal a target or a distractor item. Each trial included an arrow cue and an abrupt onset cue, and both expected cue validities and cue-character SOAs were varied factorially. The results showed that, in general, abrupt onsets captured attention more effectively than either central or peripheral arrow cues. However, tradeoffs among separate cue effects indicated that the power of abrupt onsets to capture attention automatically could be overridden by a high-validity spatial cue presented in advance of the onset character. Tradeoffs between the effects of central and abrupt onset cues were additive, indicating that endogenous and exogenous cues have their main effects at different levels in the visual attention system. Peripheral cues and abrupt onsets showed mainly interactive effects, however, consistent with the idea that both types of cues have exogenous components that affect a common pool of attentional resources.
\end{abstract}

Attention is commonly viewed as a covert orienting response that might be followed by overt movements of the eyes, head, or body. The speed of an attentional movement apparently can be much greater than that of an overt movement, as attention is capable of reallocation within the duration of a single eye fixation (see, e.g., Tsal, 1983). One metaphor commonly invoked is that of a spotlight (see, e.g., Posner, 1980), in which attention is viewed as a pool of processing resources that can be spread diffusely or concentrated to a narrow focus in response to some spatial cue or expectation. Stimuli occurring in or near the spotlight receive processing benefits, and those appearing away from it incur costs, relative to processing efficiency for stimuli presented when attention is in the diffuse mode.

Posner (1980) suggested that the beneficial effects of valid spatial cues in detection and discrimination tasks

The research reported here was supported by grants from the General Research Fund and Biomedical Sciences Committee of the University of Kansas, by a van Houten Fellowship from Philips Research Laboratories, Eindhoven, The Netherlands, and by a research fellowship from the Technical University of Eindhoven to the first author. H. K. is now at the State University of California at San Bernardino, and C.B.W. is at Kutzman State College in Pennsylvania. We thank Edwin Martin for his valuable suggestions regarding data analysis. We also thank Lester Krueger, Steven Yantis, J. Toby Mordkoff, and an anonymous reviewer for helpful comments on an earlier version of this paper. Reprint requests should be addressed to J. F. Juola, Department of Psychology, 426 Fraser Hall, University of Kansas, Lawrence, KS 66045 . could originate in at least two different ways. Exogenous cues alert an evolutionarily more primitive system derived from tendencies for organisms to orient toward important stimuli. Endogenous cues produce responses in higher cognitive centers involved in determining regions that are expected to contain an important object. Jonides (1981) argued that symbolic cues, such as a central arrow indicating some position in the periphery, typically evoke an endogenous response only, whereas cues that appear in the periphery near a potential target's position evoke an exogenous attentional response. Consistent with this view, Jonides showed that peripheral cues generally had more powerful benefits than central cues when they were valid indicators of the target's position, and costs were also greater for invalid peripheral cues than for invalid central cues. In addition, cost and benefit effects diminished with the expected validity of the central cue, whereas such expectations had virtually no effects on the costs and benefits of peripheral cues. Jonides's results are consistent with the view that peripheral cues attract attention more or less automatically, regardless of intention, whereas central cues evoke a voluntary response that can be modified at will, depending primarily on one's expectations of the cues' usefulness.

Other differences in cue effects include a shorter rise time and higher cost and benefit peaks for peripheral cues (Müller \& Rabbitt, 1989), and greater difficulty in disengaging attention from a peripheral cue than from a central cue to attend to a position elsewhere (Warner, 
Juola, \& Koshino, 1990). Both of these effects indicate that peripheral cues capture attention more completely than central cues, perhaps through special transient detection mechanisms that are more prevalent in peripheral vision (e.g., Bonnel \& Bertucci, 1991; Müller \& Rabbitt, 1989; Todd \& Van Gelder, 1979).

Yantis and Jonides (1984; Jonides \& Yantis, 1988) showed that abrupt onset of a potential target is a particularly powerful cue in capturing attention. They used a display containing a single unique item, which could be the target with chance probability, among a set of nonunique items. Uniqueness was defined by color, brightness, or abrupt onset, and only abrupt onsets showed benefits if the unique item was the target.

More recent research has indicated that peripheral cues and abrupt onsets might be only weakly automatic in their ability to capture attention, in that their effects can be overridden in some circumstances. Warner et al. (1990) showed that with thousands of trials of practice distributed over several days, observers could learn to allocate attention rapidly away from the location of a peripheral cue, so that a target occasionally occurring near the position of the cue would be detected with no special advantage. Similarly, Theeuwes (1991) and Yantis and Jonides (1990) found that the effects of an abrupt onset could be mitigated if attention were focused elsewhere, by another cue, before the onset occurred.

We have explored the relative effectiveness of various spatial cues in a task in which either a central arrow or a peripheral arrow was present on each trial (Koshino, Warner, \& Juola, 1992). In addition, one display character appeared abruptly, while others were "uncamouflaged" from preexisting figure-eight masks. In this way, the effectiveness of arrow cues was compared with that of onset cues directly, in situations in which both cues, only one cue, or neither indicated the position of the target character. The results showed that abrupt onsets generally had much larger costs and benefits than did either central or peripheral arrows. However, if observers were instructed (truthfully) that the arrows were more likely than the onsets to be valid indicators of the target's position, the costs and benefits were sometimes greater for the arrow cues than for the onsets. Thus it is suggested that all three types of cues can activate attention voluntarily, and voluntary attention to either central or peripheral arrows can sometimes suppress the automatic attention capturing potential of abrupt onset cues. Peripheral arrows and onsets can also attract attention automatically, although capture by abrupt onsets is more powerful than that by peripheral arrows. Despite these differences among cue effects, it remains uncertain whether exogenous and endogenous cues affect separate attentional resources operating at different levels in the visual system or whether the two types of cues activate a common pool of attentional resources (Koshino et al., 1992; Müller \& Humphreys, 1991).

Several problems exist in comparing the effectiveness of arrow cues and abrupt onsets. Onsets have the ability to call attention directly to the possible target position, but, by definition, they occur at the same time as target onset. Arrow cues cannot occur exactly at the target's position without risking some masking of the target, but they can precede target onset by a sufficient amount of time to enable attention to move to a focal point near the target's anticipated position. Another difference between central and peripheral arrow cues and abrupt onsets is that, for central arrow cues, subjects have to encode and interpret the cues before they can move their attention to the indicated location in order to find targets, whereas, for peripheral arrow cues, subjects simply attend to the cued area, although the cue is not as near the target as an abrupt onset cue is by definition. The present studies were designed, in part, to eliminate some of the spatial and temporal confoundings typically present in cuing studies.

\section{EXPERIMENTS 1 AND 2}

In the two experiments reported here, the effects of central and peripheral cues were compared with those of abrupt onsets, using a new method in which part of the abrupt onset character was turned on in advance. The elements that first appeared were ambiguous with regard to the target-distractor discrimination. In this way it was possible to manipulate the stimulus onset asynchrony (SOA) between the cue and subsequent display for abrupt onset cues (OSOA) as well as for central and peripheral arrow cues (ASOA) in order to chart independently the growth of their respective costs and benefits. The results should also be useful in deciding whether the various types of spatial cues compete for control of a common pool of processing resources, or whether attentional resources can be allocated in different ways to cues affecting different levels of the visual system.

In Experiment 1, a single central arrow cue and one abrupt onset item appeared on every trial along with two uncamouflaged items. The cues sometimes indicated the same item, and sometimes they did not, and both, only one, or neither could indicate the target's position on any trial. The overall validity of either cue $(75 \%$ or $25 \%)$ was manipulated factorially, between groups of subjects. Experiment 2 was identical to Experiment 1, except that a single peripheral arrow cue, near one character's position, was used on each trial instead of a central arrow.

\section{Method}

Subjects. Sixty-four University of Kansas undergraduate and graduate students, 38 males and 26 females, volunteered or participated for course credit. The subjects ranged from 18 to 36 years of age. All reported normal or corrected-to-normal vision. Half the subjects participated in Experiment 1, and half in Experiment 2, which was run after the first study. In both experiments, they were randomly assigned to four groups of 8 subjects each.

Apparatus and Stimuli. Stimulus presentation and data collection were controlled by a computer program on a Zenith Data Systems $286(12-\mathrm{MHz})$ computer with VGA graphics, and stimuli were presented on a Zenith Data Systems $\mathrm{Zm} 1490$ flat-screen 14-in. color monitor. The basic configuration of the stimulus display is shown in Figure 1. The plus sign appearing at the fixation point was replaced with an arrow pointing in one of four directions 
(central cue) in Experiment 1. Arrows external to the four possible target locations and pointing inward (peripheral cues) were used in Experiment 2. From a viewing distance of about $122 \mathrm{~cm}$ (maintained by use of a chinrest), the center of each digit was displaced about $2.45^{\circ}$ of visual angle $(5.2 \mathrm{~cm})$ from the central fixation cross. Each arrow subtended $.38^{\circ}$ of visual angle $(8 \mathrm{~mm})$ in length, and $.33^{\circ}(7 \mathrm{~mm})$ in width. Each digit was $.70^{\circ}(15 \mathrm{~mm})$ high and $.56^{\circ}(12 \mathrm{~mm})$ wide. The gap between the outside edge of each character and the head of the corresponding external, peripheral arrow was $42^{\circ}(9 \mathrm{~mm})$.

In order to manipulate SOA for abrupt onsets, three horizontal lines from the figure eight were used as a premask. With the subsequent addition of selected vertical lines and, in some cases, deletion of the top horizontal line, the three horizontals could be changed to either a target $(2$ or 5$)$ or a distractor $(3,6$, or 9$)$. The relative brightness of the cues and characters used can be judged from the numbers of pixels illuminated for each: 96 pixels for three horizontal lines used as premasks, between 125 and 138 pixels for the arrow cues and target and distractor numerals, and 180 pixels for the figure-eight premasks. Responses were made on a keyboard by using the left "alt" key for the target 2 and the right "control" key for the 5 .

Design. The experimental design for both experiments was a $2 \times 2 \times 2 \times 2 \times 3 \times 3$ mixed design, in which the betweensubjects factors were $2 \times 2$ combinations of expected overall validity of arrow cues and abrupt onsets $(25 \%$ or $75 \%)$, and the within-subjects factors were the actual arrow cue validity on any trial (valid or invalid), abrupt onset validity (valid or invalid), SOA between the arrows and targets (ASOA: 0,100 , or $200 \mathrm{msec}$ ), and the SOA between the abrupt onsets and targets (OSOA: 0, 100, or $200 \mathrm{msec}$ ). There were 128 trials for each combination of the two SOAs, yielding a total 1,152 trials per subject, and they were partitioned into two sessions of 576 trials each.

Procedure. The subjects were run individually in two 1-h sessions. The second session was held within a few days of the first session. The instructions for the four groups were identical, except that subjects were informed of the percentages of valid cues for their particular group. They were told to use the arrow cues and/or abrupt onset cues when the expected validity for them was $75 \%$, and to ignore them when the expected validity was $25 \%$. After instructions, the subjects were given 96 practice trials before the first session, and 48 practice trials to start the second session. The

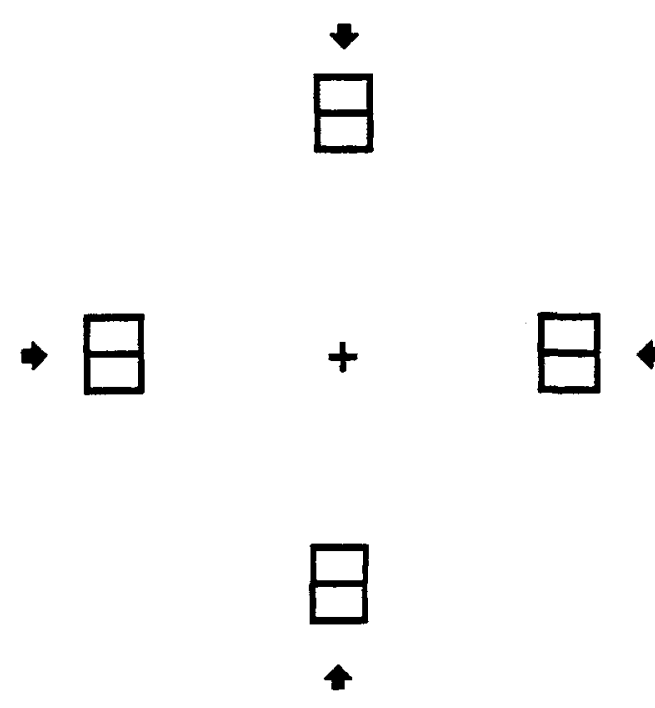

Figure 1. A representation of the display used in Experiments 1 and 2. The layout is approximately to scale.

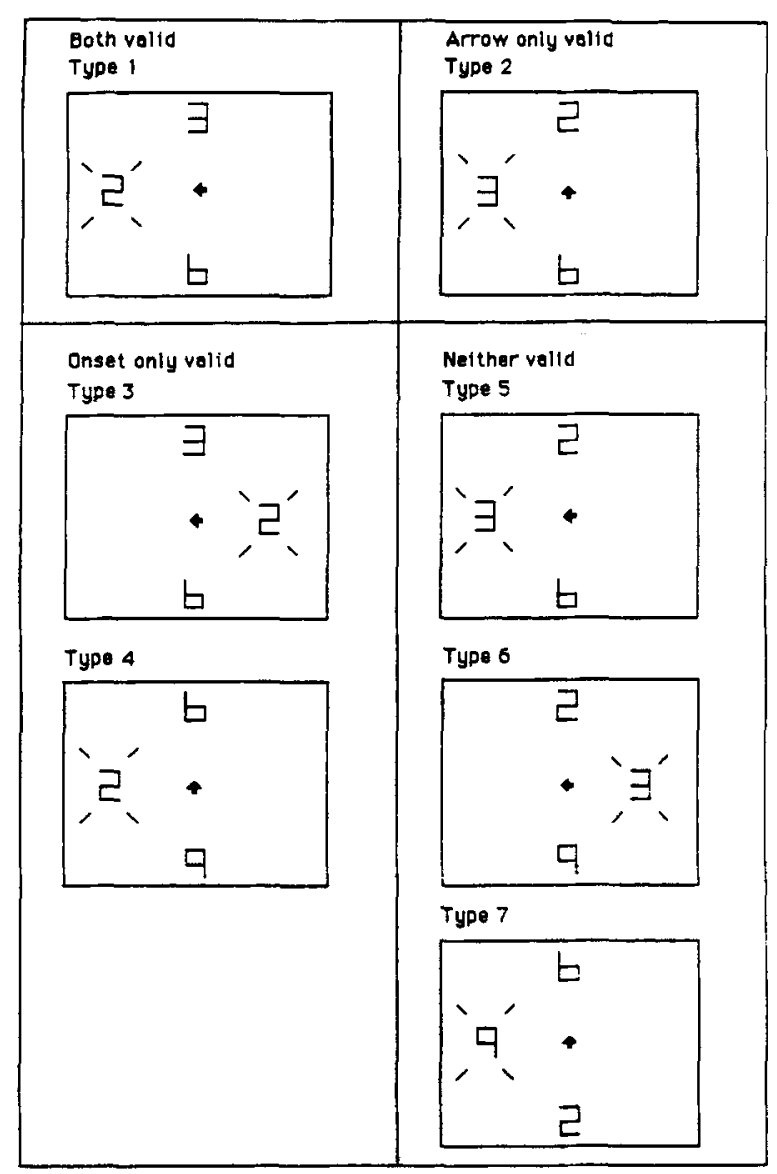

Figure 2. Seven types of stimulus patterns used in Experiments 1 and 2. Only the target " 2 " is used in these examples, although " 5 " was used as a target equally often in all conditions. Type 1 : Both the arrow and the abrupt onset are valid. Type 2: Only the arrow is valid, and the abrupt onset is invalid. Types 3 and 4: Only the abrupt onset is valid, and the arrow is invalid. Types 5,6 , and $7:$ Neither the arrow nor the abrupt onset is valid. The patterns were identical in both experiments except that central arrows were used in Experiment 1 and peripheral arrows were used in Experiment 2. The figure is from Experiment 1. (The diagonal lines in the figure indicate the abrupt onset digit and did not appear in the display.)

subsequent experimental trials were divided into nine sets for each session, consisting of 64 trials per set. At the beginning of each trial, the central fixation cross and two seven-segment figure eights were illuminated for $1 \mathrm{sec}$. The two illuminated figures were randomly presented at the top and bottom, or the left and right, of the display. Then either a central arrow cue (Experiment 1) or a peripheral arrow cue (Experiment 2) and/or a premask was illuminated according to their respective SOAs. There were seven types of stimulus patterns, as shown in Figure 2. For instance, in Type 1, in which both the arrow and abrupt onset were valid, the arrow pointed to a place where a premask was or would be presented, and either the three horizontal lines followed by the target (if OSOA $=100$ or $200 \mathrm{msec}$ ) or only the target itself (if OSOA $=0 \mathrm{msec}$ ) abruptly appeared at the cued position. All four groups of subjects were presented with examples of all seven trial types, but the relative proportions were fixed to keep the overall cue validities at either $25 \%$ or $75 \%$ in the respective groups. An example of the trial sequence for trial Type 1 (both cues valid) is shown in Figure 3. 


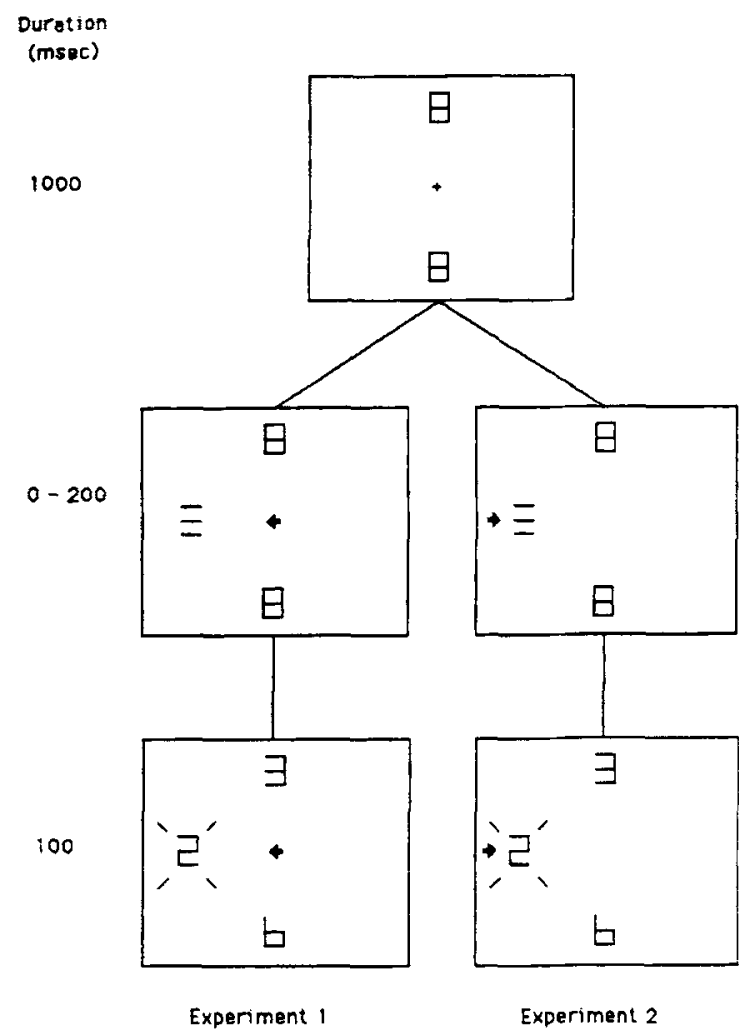

Figure 3. An example of the trial sequence. Top: Two figure-8s were presented either vertically or horizontally centered around the fixation point, and stayed on for $1,000 \mathrm{msec}$. Middle: An arrow and/or a premask for an abrupt onset were presented with either 0 , 100 , or 200 msec SOA (with the exception that no premask was used in the 0 msec onset condition-the character itself appeared abruptty). The sequence for Experiment 1 is shown on the left, and that for Experiment 2 is shown on the right. Bottom: The premask turned into a digit and the two figure-8s were replaced by two digits. The trial sequence was identical for both experiments except for the positions of the arrow cues.

The subjects were asked to respond as quickly as possible and to try to keep their error rates below 5\%. Following an error, the computer emitted a tone, and a buffer trial was inserted. The data from buffer trials were not included in any analysis. At the end of each set of 64 trials, the subjects were given feedback about their mean response time (RT) and error rate and allowed a short rest break before continuing.

\section{Results and Discussion}

Response time data. Mean RT data for correct responses in all conditions of both experiments are shown in Table 1. After collapsing the RT data over both ASOA and OSOA, a preliminary analysis was made. These collapsed data are shown in Figure 4.

In Group 1 (in which both arrow and onset cues were valid $25 \%$ of the time: $25 \mathrm{a} / 25 \mathrm{o}$ ), differences in RTs among cue validity conditions were not very large. The average difference in RTs for valid and invalid arrow cue trials was $7 \mathrm{msec}$ for the central arrows used in Experiment 1 , and this difference was $6 \mathrm{msec}$ for the peripheral arrows in Experiment 2. The mean RT difference between valid and invalid onset cue trials was $30 \mathrm{msec}$ in Experiment 1 and $49 \mathrm{msec}$ in Experiment 2.

In Group 2 (in which arrows were valid $25 \%$ of the time, and onsets were $75 \%$ valid: $25 \mathrm{a} / 75 \mathrm{o}$ ), the central arrow cues had no measurable effect (mean RT difference between valid and invalid cue trials $=0 \mathrm{msec}$ ), but mean RT for valid peripheral arrow cue trials was $18 \mathrm{msec}$ less than that for invalid arrow cues. In contrast, the validity effect for onset cues was $184 \mathrm{msec}$ and $188 \mathrm{msec}$ in Experiments 1 and 2, respectively.

The data from Group 3 (arrow validity $=75 \%$, and onset validity $=25 \%: 75 \mathrm{a} / 250$ ) stand in marked contrast, however: the central arrow cues used in Experiment 1 actually had a slightly larger effect than did the abrupt onsets ( 57 vs. 50 msec for the respective differences between mean RTs for valid and invalid arrow and onset cues). The data from Experiment 2 were similar: the validity effect was $76 \mathrm{msec}$ for peripheral arrow cues and $74 \mathrm{msec}$ for abrupt onsets.

In Group 4 (both arrows and onsets valid $75 \%$ of the time: $75 \mathrm{a} / 75 \mathrm{o}$ ), onset validity again had a larger effect than arrow validity had; the mean RT differences between valid and invalid trials were $22 \mathrm{msec}$ for central arrow cues and $209 \mathrm{msec}$ for onset cues in Experiment 1, and the mean RT differences were $75 \mathrm{msec}$ for peripheral arrow cues and $223 \mathrm{msec}$ for onset cues in Experiment 2.

The data were subjected to a 4 (subject group) $\times 2$ (arrow cue validity) $\times 2$ (abrupt onset cue validity) mixed analysis of variance (ANOVA) for each experiment separately. The group main effects were nonsignificant, but there were significant effects of both arrow cue validity $[F(1,28)=21.41, p<.001$, for $\mathrm{Ex}$ periment 1 , and $F(1,28)=116.2, p<.001$, for Experiment 2] and abrupt onset validity $[F(1,28)=286.7, p<$ .001 , and $F(1,28)=115.8, p<.001$, for Experiments 1 and 2 , respectively]. In addition, all two-way interactions were significant in both experiments, as were the respective three-way interactions of group $\times$ arrow validity $\times$ onset validity $[F(3,28)=3.17, p<.05$, and $F(3,28)=7.99, p<.05]$. These results show that the overall validities of arrow and onset cues were effective in manipulating the size of the cue validity effects. However, central arrow cues and even peripheral arrow cues could be effectively ignored when they were generally invalid, but onset cues could not. The effects of onset cues were also larger overall than those of both types of arrow cues, except for Group 3, in which subjects knew that the arrows were generally valid and the abrupt onsets were generally not. An analysis of the SOA effects follows in the next section, in which the overall RTs were converted to costs plus benefits.

Costs plus benefits. Costs plus benefits were determined by subtracting mean RTs on valid cue trials from those on invalid cue trials for each cue separately, collapsing across validity levels of the other cue. The costs plus benefits are plotted against arrow SOA (ASOA) for 
Table 1

Mean Response Times (in Milliseconds) and Percentages of Errors for All Conditions of Experiments 1 and 2

\begin{tabular}{|c|c|c|c|c|c|c|c|c|c|c|c|c|c|c|c|c|c|c|}
\hline \multirow[b]{3}{*}{ Cues } & \multicolumn{6}{|c|}{0 -msec OSOA } & \multicolumn{6}{|c|}{ 100-msec OSOA } & \multicolumn{6}{|c|}{ 200-msec OSOA } \\
\hline & \multicolumn{2}{|c|}{$\begin{array}{c}0-\mathrm{msec} \\
\text { ASOA }\end{array}$} & \multicolumn{2}{|c|}{$\begin{array}{l}100-\overline{m s e c} \\
\text { ASOA }\end{array}$} & \multicolumn{2}{|c|}{$\begin{array}{c}200-\mathrm{msec} \\
\text { ASOA }\end{array}$} & \multicolumn{2}{|c|}{$\begin{array}{l}0 \text {-msec } \\
\text { ASOA }\end{array}$} & \multicolumn{2}{|c|}{$\begin{array}{c}\text { 100-msec } \\
\text { ASOA }\end{array}$} & \multicolumn{2}{|c|}{$\begin{array}{c}200-\mathrm{msec} \\
\text { ASOA }\end{array}$} & \multicolumn{2}{|c|}{$\begin{array}{l}0 \text {-msec } \\
\text { ASOA }\end{array}$} & \multicolumn{2}{|c|}{$\begin{array}{c}100-\mathrm{msec} \\
\text { ASOA }\end{array}$} & \multicolumn{2}{|c|}{$\begin{array}{c}200-\mathrm{msec} \\
\text { ASOA }\end{array}$} \\
\hline & $\mathrm{RT}$ & $\mathrm{PE}$ & $\mathrm{RT}$ & $\overline{\mathrm{PE}}$ & $\mathrm{RT}$ & $\mathrm{PE}$ & $\mathrm{RT}$ & $\mathrm{PE}$ & RT & $\mathrm{PE}$ & RT & PE & $\mathrm{RT}$ & PE & RT & $\mathrm{PE}$ & RT & $\mathrm{PE}$ \\
\hline \multicolumn{19}{|c|}{ Experiment 1} \\
\hline \multicolumn{19}{|c|}{ Group l (25a/25o) } \\
\hline avov & 515 & 2.4 & 512 & 2.4 & 520 & 0.7 & 536 & 1.5 & 518 & 3.1 & 516 & 1.5 & 530 & 3.1 & 528 & 3.1 & 529 & 6.4 \\
\hline avoi & 562 & 4.0 & 584 & 5.5 & 552 & 5.2 & 563 & 2.4 & 553 & 9.6 & 538 & 6.4 & 543 & 7.7 & 541 & 7.0 & 536 & 3.9 \\
\hline aiov & 527 & 1.5 & 509 & 2.2 & 515 & 4.6 & 534 & 7.9 & 530 & 3.0 & 547 & 3.9 & 540 & 3.9 & 524 & 3.7 & 532 & 5.5 \\
\hline aioi & 561 & 4.9 & 561 & 5.4 & 556 & 4.9 & 555 & 4.6 & 555 & 4.4 & 564 & 6.5 & 572 & 6.4 & 565 & 4.1 & 561 & 5.0 \\
\hline \multicolumn{19}{|c|}{ Group $2(25 \mathrm{a} / 75 \mathrm{o})$} \\
\hline avov & 503 & 2.4 & 480 & 1.6 & 490 & 0.7 & 492 & 3.0 & 482 & 3.9 & 488 & 3.1 & 481 & 6.1 & 487 & 6.2 & 483 & 5.4 \\
\hline avoi & 667 & 8.5 & 673 & 11.1 & 667 & 7.1 & 681 & 10.2 & & 10.2 & 696 & 12.5 & 68 & 6.2 & & 12.6 & & 7.9 \\
\hline aiov & 494 & 1.7 & 486 & 1.9 & 496 & 2.0 & 473 & 2.2 & 4 & 2.1 & 487 & 3. & 48 & 3 & 485 & & 92 & 3.9 \\
\hline aioi & 672 & 11.9 & 686 & 10.1 & 674 & 9.4 & 675 & 8.6 & 674 & 10.4 & 659 & 11.7 & 664 & 7.9 & 665 & 8.6 & 670 & 13.2 \\
\hline \multicolumn{19}{|c|}{ Group $3(75 \mathrm{a} / 250)$} \\
\hline avov & 536 & 2.4 & 497 & 4.0 & 507 & 4.2 & 548 & 2.4 & 531 & 3.1 & 524 & 1.5 & 570 & 3.1 & 548 & 2.4 & 565 & 7.1 \\
\hline avoi & 617 & 4.6 & 581 & 5.2 & 535 & 4.2 & 604 & 6.5 & 5 & 6. & 548 & 4.7 & 59 & 5.9 & & 4.0 & 57 & 6.2 \\
\hline aiov & 567 & 2.2 & 576 & 3.9 & 589 & 1.5 & 564 & 4.7 & 56 & 5.5 & 587 & 5.5 & 601 & 7.1 & 60 & 10.1 & 600 & 4.6 \\
\hline aioi & 655 & 7.0 & 641 & 7.7 & 648 & 8.6 & 643 & 7.9 & 643 & 5.5 & 639 & 6.4 & 645 & 7.0 & 625 & 6.2 & 647 & 16.6 \\
\hline Group 4 & $75 \mathrm{a} / 7$ & & & & & & & & & & & & & & & & & \\
\hline avov & 498 & 1.4 & 491 & 2.1 & 486 & 1.2 & 490 & 1.7 & 488 & 3.9 & 487 & 6.2 & 483 & 3.9 & 487 & 6.6 & 479 & 5.1 \\
\hline avoi & 708 & 8.6 & 665 & 14.9 & 63 & 3.1 & 736 & 10.1 & & 9.5 & 67 & 8.5 & 74 & 8.5 & & & & 5.5 \\
\hline aiov & 522 & 5.4 & 504 & 3.1 & 519 & 0 & 494 & 3.1 & 50 & 4.0 & 50 & 3. & 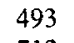 & & & & & 2.4 \\
\hline aioi & 749 & 14.9 & 729 & 7.2 & 710 & 8.7 & 739 & 8.2 & 720 & 7.7 & 707 & 6.1 & 712 & 5.2 & 685 & 9.1 & 718 & 5.5 \\
\hline & & & & & & & & & & & & & & & & & & \\
\hline Groum 1 & 25912 & & & & & & & & & & & & & & & & & \\
\hline avov & 609 & 3.1 & 589 & 1.5 & 565 & 0.7 & 584 & 3.9 & 579 & 6.2 & 571 & 3.0 & 592 & 5.4 & 580 & 6.2 & 568 & 2.2 \\
\hline avoi & 626 & 13.4 & 613 & 7.1 & 608 & & 651 & 11.9 & & & 616 & 2.2 & & 8.7 & 66 & 3.0 & 634 & 7.1 \\
\hline aiov & 582 & 2.4 & 594 & 0.7 & 597 & 1.5 & 583 & 0 & 57 & 1.5 & 592 & 0.7 & 583 & 1.5 & 59 & 2.2 & 594 & 2.2 \\
\hline aioi & 626 & 4.2 & 641 & 3.9 & 639 & 4.1 & 630 & 3.5 & 634 & 2.5 & 643 & 4.2 & 635 & 3.9 & 637 & 3.6 & 637 & 4.1 \\
\hline Group 2 & $25 \mathrm{a} / 7$ & & & & & & & & & & & & & & & & & \\
\hline avov & 512 & 3.1 & 502 & 0 & 494 & 0 & 523 & 3.9 & 517 & 3.1 & 509 & 1.6 & 523 & 8.6 & 525 & 2.2 & 519 & 3.0 \\
\hline avoi & 751 & 12.4 & 640 & 7.1 & 617 & 11.9 & 727 & 14.9 & 67 & 12.0 & 6 & & 72 & 15.7 & 69 & 12.6 & 86 & 12.5 \\
\hline aiov & 508 & 0.7 & 514 & 1.2 & 532 & 1.0 & 512 & 1.7 & 51 & 1.4 & 51 & & & 2.6 & & & 518 & 3.0 \\
\hline aioi & 708 & 10.1 & 725 & 7.9 & 721 & 6.2 & 732 & 10.4 & 704 & 12.6 & 744 & 7.9 & 707 & 10.2 & 716 & 10.7 & 736 & 10.2 \\
\hline Group 3 & & & & & & & & & & & & & & & & & & \\
\hline & 573 & 1.5 & 562 & 0.7 & 552 & 1.5 & 556 & 3.7 & 561 & 1.5 & 552 & 2.4 & 569 & 4.6 & 54 & 5.5 & 539 & 2.4 \\
\hline avoi & 637 & 15.6 & 580 & 8.6 & 545 & 6.7 & 656 & 10.4 & 59 & 6.0 & 571 & 6. & 649 & 10.4 & 60 & 7.5 & 576 & 5.6 \\
\hline aiov & 601 & 1.6 & 621 & 3.1 & 641 & 3.9 & 576 & 4.7 & 616 & 2.2 & 609 & 4.7 & 580 & 2.4 & 586 & 4.7 & 597 & 5.5 \\
\hline aioi & 706 & 9.4 & 697 & 5.5 & 705 & 11.1 & 694 & 5.4 & 711 & 8.6 & 722 & 4.7 & 686 & 8.0 & 686 & 7.9 & 750 & 11.0 \\
\hline Group 4 & $75 \mathrm{a} / 7$ & & & & & & & & & & & & & & & & & \\
\hline avov & 512 & 4.1 & 483 & 2.0 & 475 & 1.0 & 499 & 3.9 & 500 & 1.9 & 477 & 5.0 & 475 & 2.7 & 45 & 3.6 & 449 & 4.4 \\
\hline avoi & 711 & 23.5 & 645 & 7,1 & 574 & 5.4 & 734 & 18.1 & 712 & 6.9 & 636 & 3.1 & 720 & 13.4 & 67 & & 648 & 7.9 \\
\hline aiov & 513 & 0 & 537 & 0.7 & 633 & 6.2 & 504 & 0.7 & 533 & 3.9 & 563 & 7.1 & 476 & 0.7 & 474 & & 492 & 2.2 \\
\hline aioi & 762 & 47.0 & 851 & 14.1 & 801 & 16.5 & 745 & 11.1 & 750 & 10.2 & 820 & 20.5 & 754 & 10.4 & 744 & 9.4 & 780 & 12.6 \\
\hline
\end{tabular}

Note-ASOA, central arrow cue stimulus onset asynchrony; OSOA, abrupt onset cue stimulus onset asynchrony; a, arrow cue; o, onset cue; $v$, valid cue; $i$, invalid cue.

each level of abrupt onset SOA (OSOA) in Figure 5. Within each panel of Figure 5, the data are shown for the four groups separately. The data are summarized further by collapsing across subject groups and SOAs in Table 2 . In general, the data show that when the expected validity of the central arrow is low [Groups $1(25 \mathrm{a} / 25 \mathrm{o})$ and $2(25 \mathrm{a} / 750)]$, arrow cues have little or no costs plus benefits $(\bar{X}=4 \mathrm{msec}$ in Experiment 1 , and $\bar{X}=12 \mathrm{msec}$ in Experiment 2). The same is not true for the effects of abrupt onsets, as their costs plus benefits fall above the zero line in groups with low expected validity for onset cues [Groups $1(25 \mathrm{a} / 25 \mathrm{o})$ and $3(75 \mathrm{a} / 25 \mathrm{o}) ; \bar{X}=40 \mathrm{msec}$ in Experiment 1, and $\bar{X}=62 \mathrm{msec}$ in Experiment 2]. Costs plus benefits increased when expected cue validity increased from $25 \%$ to $75 \%$ by an average of $36 \mathrm{msec}$ for central arrows and $156 \mathrm{msec}$ for onsets in Experiment 1, and by $64 \mathrm{msec}$ for peripheral arrows and $144 \mathrm{msec}$ for onsets in Experiment 2 (see Table 2). Thus, even though the onset cues can capture attention automatically, by producing cost-benefit effects when they have chance validity, they do not always capture attention completely. That is, onset cues produced an even larger gain in costs plus benefits when their validities increased from $25 \%$ to $75 \%$ than did the arrow cues. The bottom-up attention- 


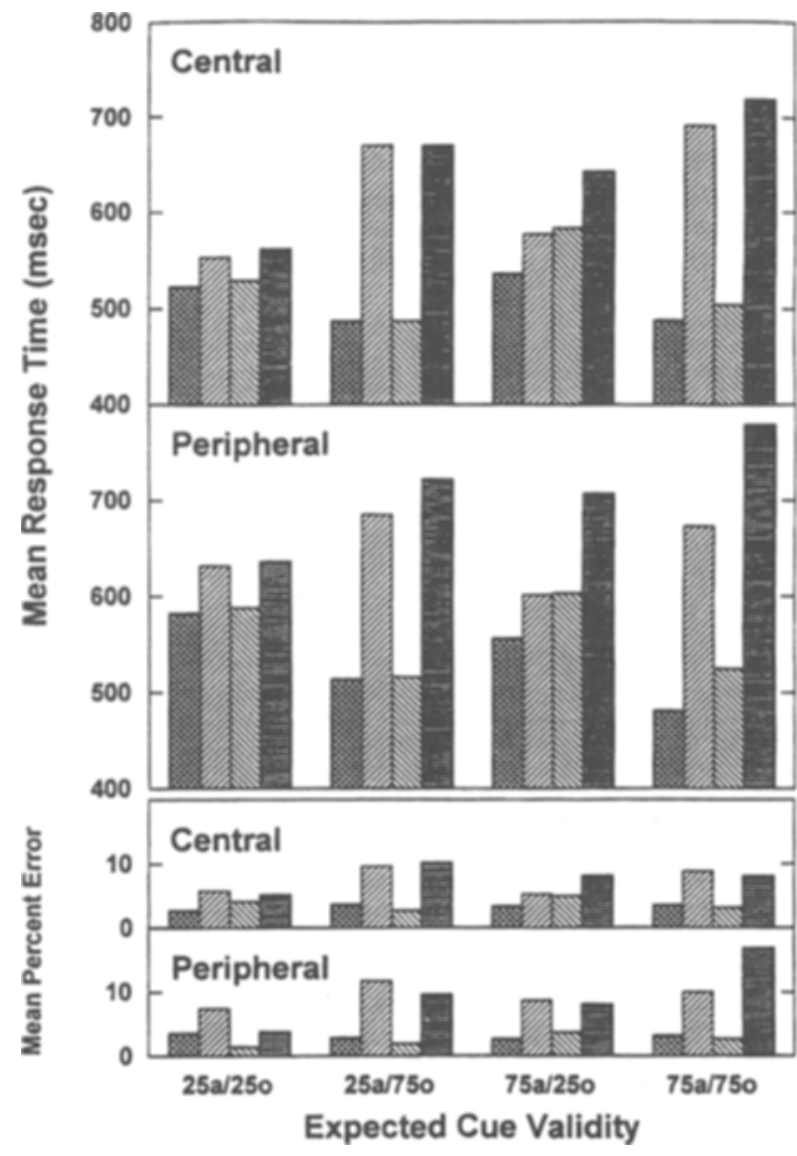

Figure 4. Mean response time in Experiments 1 (central) and 2 (peripheral) plotted against the expected cue validity for each of four groups of subjects (e.g., 25a/250 = Group 1, for which $25 \%$ of the arrow cues and $25 \%$ of the abrupt onsets were likely to indicate targets). The data are plotted separately within each group for respective (left-to-right columns) trials on which both cues indicated the target position, only the arrow cue was valid, only the onset cue was valid, or neither cue was valid.

capturing potential of an abrupt onset cue apparently can be augmented or diminished by top-down controls that voluntarily maintain focus on the onset cue when it is likely to be valid or redirect it from the cued location when the onset is unlikely to be a valid cue.

In order to compare the relative effects of arrow and onset cues ${ }^{1}$ across the four groups, the costs-plus-benefits data from each experiment were subjected to a 2 (expected arrow cue validity) $\times 2$ (expected abrupt onset cue validity) $\times 2$ (arrow vs. onset cues) $\times 3$ (arrow SOA: ASOA) $\times 3$ (onset SOA: OSOA) mixed ANOVA. There were significant main effects of expected cue validity in Experiment 1: $F(1,28)=13.21, p<.01$, for expected central arrow cue validity, and $F(1,28)=71.77, p<$ .001 , for expected abrupt onset cue validity. Similarly, in Experiment 2, expected cue validity was significant for peripheral arrows $[F(1,28)=13.48, p<.01]$ and for abrupt onsets $[F(1,28)=35.05, p<.001]$. Costs plus benefits were also greater for onsets than for central arrows in Experiment $1[F(1,28)=118.94, p<.001]$, and they were greater for onsets than for peripheral arrows in Experiment $2[F(1,28)=44.93, p<.001]$. In Experiment 1 , mean overall costs plus benefits increased with OSOA $[F(2,56)=3.41, p<.05]$, whereas in Experiment 2 , costs plus benefits increased with ASOA $[F(2,56)=23.29, p<.001]$.

There were a number of significant higher order interactions involving expected arrow or onset cue validities, which were between-group manipulations. Therefore, in order to examine these interactions more closely, and also to compare the effects of central as opposed to peripheral arrow cues, the data from each group were taken from both experiments and analyzed in separate 2 (experiment) $\times 2$ (arrow vs. onset cue) $\times 3($ ASOA $) \times 3$ (OSOA) mixed ANOVAs. In the following discussion, only those interactions involving experiment will be presented, since the others are redundant with effects discussed earlier. For Group 1, there was a significant three-way interaction for experiment $\times$ cue $\times$ OSOA $[F(2,28)=9.25, p<.01]$, reflecting the fact that costs plus benefits decreased with OSOA for onset cues and increased with OSOA for arrow cues in Experiment 1, whereas these effects were reversed in Experiment 2.

In Group 2, there was a significant interaction among all four variables $[F(4,56)=2.89, p<.05]$. In Experiment 1 , there was virtually no effect of either OSOA or ASOA, whereas there were striking tradeoffs between the effects of peripheral arrows and abrupt onsets in Experiment 2 as a function of ASOA. These tradeoffs diminished with increasing OSOA.

Group 3 showed an experiment $\times$ cue $\times$ OSOA interaction $[F(2,28)=6.80, p<.01]$, like that found in Group 1. In addition, there was an experiment $\times$ cue $\times$ ASOA interaction $[F(2,28)=3.84, p<.05]$, indicating that the tradeoffs between the effects of peripheral arrow cues and abrupt onsets across ASOA in Experiment 2 were stronger than those among arrows and onsets in Experiment 1. Finally, Group 4 showed the same fourway interaction as did Group $2[F(4,56)=2.70, p<$ $.05]$, with a similar interpretation.

These interactions among experiments support two general conclusions: (1) It is easier to ignore central arrow cues than peripheral arrow cues when the arrow cues are generally invalid. (2) It is easier to separate attentional allocation among central arrows and abrupt onsets than among peripheral arrows and abrupt onsets when the arrows are generally valid. That is, it appears that two types of peripheral cues (peripheral arrows and abrupt onsets) occurring on a single trial are more likely to command the same resources, and thus to produce tradeoffs among cue effects, than are a central arrow and an abrupt onset cue. In other words, central arrow cues and abrupt onsets seem to be generally independent in their effects as measured by costs plus benefits, whereas peripheral arrows and abrupt onsets appear to affect a common attentional system, producing dependencies in their effects. 


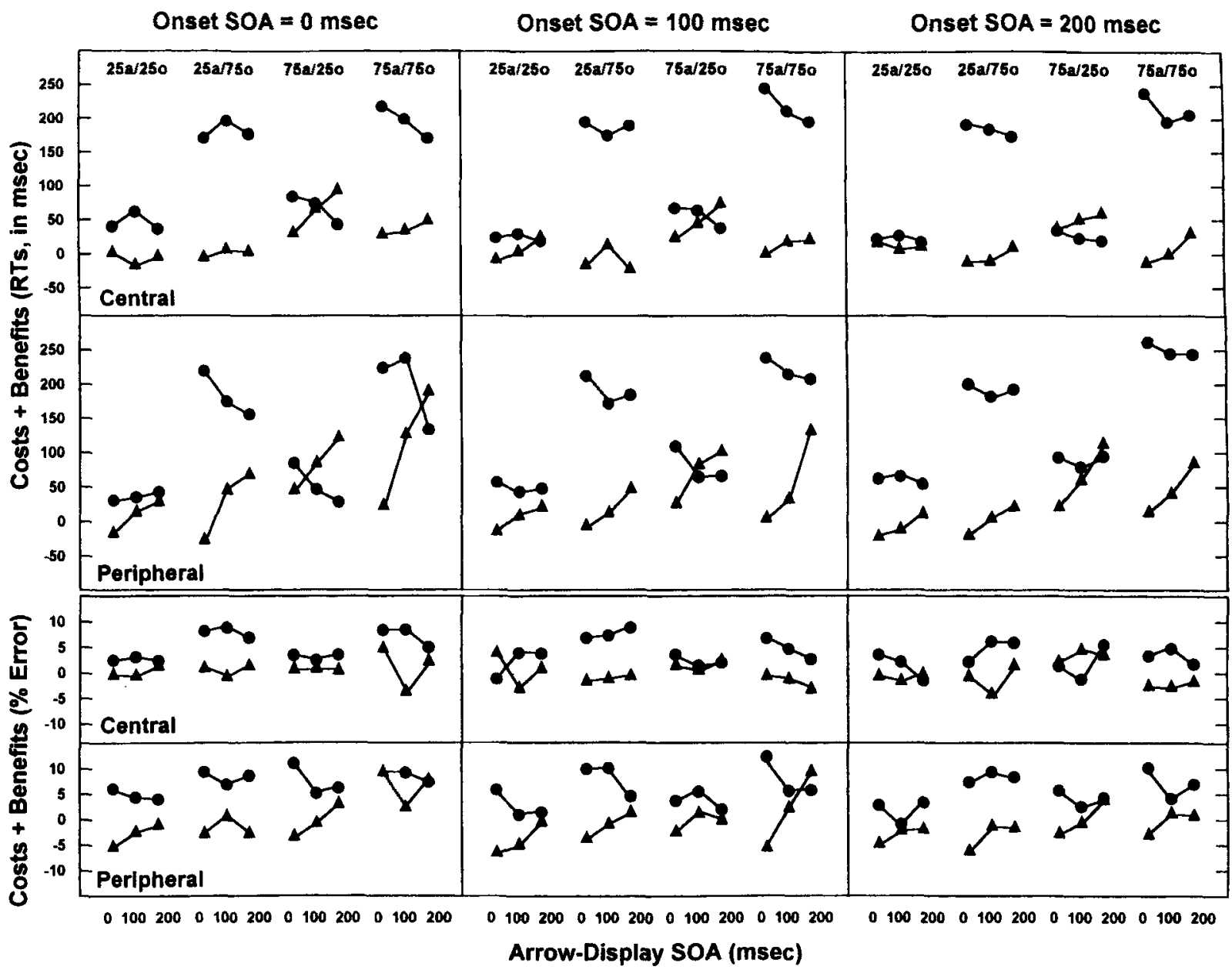

Figure 5. Mean costs plus benefits in Experiments 1 (central) and 2 (peripheral) plotted against arrow-display SOA for each subject group. The three panels show the data for each onset SOA separately. The triangles represent data for arrow cues, and the circles represent data for abrupt onset cues.

As a test of independence between the two types of cues used in both experiments, the costs plus benefits were determined for each cue separately for trials on which the other cue was either valid or invalid. If the cues have independent effects, the costs plus benefits of any cue should not depend on the validity of the other cue. This could be the case, for example, if exogenous (onset) cues affected an attentional resource pool available earlier in visual processing than that tapped by endogenous (central arrow) cues. On the other hand, if both cues affect allocation of a common pool of resources, the effect of either cue should depend on the validity of the other. Such dependencies should be visible in the results of Experiment 2, since abrupt onsets and peripheral arrow cues should always elicit exogenous attentional responses, and they should both attract attention endogenously as well when their validities are high.

The data shown in Table 3 are the mean costs plus benefits in the RT data for each cue in all groups of both experiments, partitioned into trials on which the other cue was either valid or invalid. As can be seen in the table, this partitioning results in small differences in the data for Experiment 1, but large differences in the results for most groups in Experiment 2. In Experiment 1, the mean costs plus benefits were $8 \mathrm{msec}$ less for arrow cues, when the onset cues were valid rather than invalid, and the costs plus benefits for onset cues were $9 \mathrm{msec}$ less on trials with valid central arrow cues as opposed to invalid arrow cues. In Experiment 2, these differences were $38 \mathrm{msec}$ for arrow cues and $41 \mathrm{msec}$ for onset cues. These results are consistent with the relative independence of cue effects for central arrows and abrupt onsets (Experiment 1), whereas the common attentional effects of peripheral arrow and abrupt onset cues (Experiment 2) is demonstrated by their lack of independence.

Error data. The mean error rate was about $4.9 \%$ in Experiment 1 and about $6.1 \%$ in Experiment 2. The correlations between mean RTs and error proportions across all 36 conditions ( 2 levels of arrow cue validity, 2 levels of onset cue validity, and the $3 \times 3$ combinations 
Table 2

Costs Plus Benefits for Response Times (in Milliseconds) and for Error Rates (Percentages) for Arrow and Onset Cues in Experiments 1 and 2, Averaged Across Cue-Display Stimulus Onset Asynchronies, for Conditions in Which the Cues Were Valid Either $25 \%$ or $75 \%$ of the Time

\begin{tabular}{|c|c|c|c|c|}
\hline \multirow[b]{3}{*}{ Cue Type } & \multicolumn{4}{|c|}{ Costs Plus Benefits } \\
\hline & \multicolumn{2}{|c|}{$25 \%$ Cue Validity } & \multicolumn{2}{|c|}{$75 \%$ Cue Validity } \\
\hline & for RTs & for Errors & for RTs & for Errors \\
\hline \multicolumn{5}{|c|}{ Experiment 1} \\
\hline Arrow & 4 & 0.1 & 40 & 0.9 \\
\hline Onset & 40 & 2.3 & 196 & 6.0 \\
\hline \multicolumn{5}{|c|}{ Experiment 2} \\
\hline Arrow & 12 & -2.2 & 76 & 2.0 \\
\hline Onset & 62 & 4.2 & 206 & 9.7 \\
\hline
\end{tabular}

of arrow and onset SOAs) were significant for all groups in both experiments. The $r$ values for Groups $1-4$ respectively were $.46, .89, .37$, and .74 for Experiment 1 , and they were $.46, .86, .48$, and .69 for Experiment 2 (all $p s<.05)$. Means of raw error rates are shown in Table 1 , and the error data collapsed across ASOA and OSOA are shown in Figure 4.

The error data from both experiments were subjected to a 2 (expected central cue validity) $\times 2$ (expected onset cue validity) $\times 2$ (central cue validity) $\times 2$ (abrupt onset validity $\times 3(\mathrm{ASOA}) \times 3(\mathrm{OSOA})$ mixed ANOVA. There was a main effect of abrupt onset cue validity $[F(1,28)=$ $17.11, p<.001$, in Experiment 1, and $F(1,28)=45.60$, $p<.001$, in Experiment 2]. Error rates were $3.5 \%$ on valid onset cue trials in Experiment 1 and $2.7 \%$ on valid onset cue trials in Experiment 2. The error rates for invalid onset cue trials were 7.6\% and 9.5\% for Experiments 1 and 2 , respectively. The validity of arrow cues had no effect on error rates in either study. A three-way interaction with no obvious interpretation was obtained in Experiment 1 for central arrow cue validity $\times$ abrupt onset cue validity $\times \operatorname{ASOA}[F(2,56)=3.87, p<.05]$. In Experiment 2, there was a simple main effect of ASOA, showing that more errors were made at the 0 -msec arrow SOA $(7.7 \%)$ than at 100 - or $200-\mathrm{msec}$ ASOA $(5.2 \%$ and $5.5 \%$, respectively) $[F(2,56)=20.23, p<.001]$. However, all factors entered into a significant six-way interaction $[F(4,112)=3.72, p<.01]$, which was not readily interpretable.

The costs plus benefits were found for the error data in the same way as they were for the RT data, and the results are shown in Figure 5. The error data were examined for cue independence in the same way as were the RT data: by comparing the costs plus benefits of any cue for valid versus invalid conditions for the other cue (see Table 3). The results showed that the costs plus benefits for any cue differed little in Experiment 1, depending on the validity of the other cue. The costs plus benefits were the same for central arrow cues whether the onset cue was valid or invalid, and they were $.1 \%$ higher for onset cues when the central arrow was invalid as opposed to valid. In Experiment 2, the costs plus benefits differences were still small, but larger by an order of magnitude. Costs plus benefits were $.8 \%$ higher for peripheral arrow cues when the onset cue was invalid, and the costs plus benefits for the onset cues was. $7 \%$ higher when the peripheral arrow was invalid. These results parallel those of the RT data; the effects of central arrow cues and abrupt onsets are relatively independent as measured by costs and benefits in the error data, whereas the costs plus benefits of both peripheral arrow cues and abrupt onsets are higher if the other cue is invalid as opposed to valid, indicating a lack of independence in their attentional effects.

\section{GENERAL DISCUSSION}

The two experiments presented here were designed to compare three types of spatial cues in terms of their effects, and the relative time courses of these effects, in a

Table 3

Mean Costs Plus Benefits for Response Times (in Milliseconds) and for Error Rates (Percentages) for Each Cue Conditionalized on the Validity of the Other Cue

\begin{tabular}{|c|c|c|c|c|c|c|c|c|}
\hline \multirow[b]{4}{*}{ Group } & \multicolumn{8}{|c|}{ Costs Plus Benefits } \\
\hline & \multicolumn{4}{|c|}{ Arrow } & \multicolumn{4}{|c|}{ Onset } \\
\hline & \multicolumn{2}{|c|}{ Onset Cue Valid } & \multicolumn{2}{|c|}{ Onset Cue Invalid } & \multicolumn{2}{|c|}{ Arrow Cue Valid } & \multicolumn{2}{|c|}{ Arrow Cue Invalid } \\
\hline & for RTs & for Errors & for RTs & for Errors & for RTs & for Errors & for RTs & for Errors \\
\hline \multicolumn{9}{|c|}{ Experiment 1} \\
\hline$I(25 a / 250)$ & 6 & 1.3 & 8 & -0.6 & 29 & 3.1 & 32 & 1.1 \\
\hline $2(25 \mathrm{a} / 75 \mathrm{o})$ & 0 & -1.0 & -1 & 0.6 & 184 & 6.0 & 184 & 7.6 \\
\hline $3(75 a / 250)$ & 47 & 2.0 & 66 & 2.8 & 40 & 2.2 & 60 & 3.1 \\
\hline $4(75 a / 75 o)$ & 16 & -0.5 & 28 & -0.7 & 203 & 5.2 & 215 & 5.0 \\
\hline \multicolumn{9}{|c|}{ Experiment 2} \\
\hline $1(25 \mathrm{a} / 25 \mathrm{o})$ & 6 & -1.6 & 4 & -3.6 & 49 & 3.8 & 48 & 2.4 \\
\hline $2(25 \mathrm{a} / 750)$ & 2 & -1.2 & 37 & -2.1 & 159 & 8.9 & 205 & 7.6 \\
\hline $3(75 a / 250)$ & 47 & 1.0 & 105 & -0.6 & 4 & 5.9 & 103 & 4.3 \\
\hline $4(75 a / 75 o)$ & 44 & -0.5 & 106 & 6.9 & 192 & 6.8 & 254 & 14.2 \\
\hline
\end{tabular}


character recognition task. Previous research has shown that valid spatial cues can provide benefits and invalid cues can incur costs in recognition times when compared with a no-cue control condition. The spotlight metaphor commonly invoked describes attention as a concentrated beam of processing resources that speeds visual processing when a target character is enveloped by the spotlight. When the spotlight is misdirected, however, time is lost when the spotlight must be enlarged, moved, or both until the target once again is included within the beam of attention.

The present studies showed large cuing effects in a character recognition task, consistent with the view that a target character can be identified more quickly if focal attention is brought to bear on the target's position before or soon after the target's onset. Central arrow cues apparently resulted in only controlled, endogenous responses, whereas peripheral arrow cues produced both controlled and exogenously triggered automatic attentional responses, as indicated by their relative effects when subjects were encouraged to ignore them. In contrast, abrupt onset cues showed larger controlled and automatic effects than did either central or peripheral arrows in most conditions. These results confirm the idea that the abrupt onset of a potential target is a particularly salient attentional cue, perhaps because the spatiotemporal continuity of the onset cues and targets used in the present studies cannot be matched by an adjacent, peripheral arrow cue (Yantis \& Gibson, 1994; Yantis \& Jonides, 1984). Although both automatic and voluntary attentional responses were larger overall for abrupt onset cues than for central or peripheral arrows, the arrows could be more effective than the onset cues if their expected validity was higher than that of the onsets and if the arrows preceded the display (see also Theeuwes, 1991; Yantis \& Jonides, 1990).

Interactions among the effects of different cues presented on the same trial are also consistent with the view that central arrow cues elicit primarily endogenous attentional responses, whereas peripheral arrow and abrupt onset cues command attention exogenously as well as endogenously. If all three types of cues affect a common pool of attentional resources that can be triggered by an exogenous or endogenous cue, we would expect to find similar validity and SOA effects for all cue types. If, on the other hand, attention is not a unitary resource but exists at several levels in the visual system, reserving different resources for early and late visual processes, there is reason to believe that validity effects for different cue types should be additive, and SOA effects need not show neat tradeoff relations between cues.

An examination of Figure 5 shows that tradeoffs existed between the pairs of cues shown on all trials. That is, the costs plus benefits for arrow cues generally increased with ASOA and decreased with OSOA, and the costs plus benefits for onset cues generally increased with OSOA and decreased with ASOA. These tradeoff relations were stronger in Experiment 2, as would be ex- pected if the cues were trading off in their control of a common resource pool. A similar conclusion can be reached from the costs plus benefits data shown in Table 3: the effects of central arrow and abrupt onset cues are relatively independent of each others' validities, whereas the costs plus benefits of either peripheral arrows or abrupt onsets are larger when the other cue is invalid than when it is valid. This dependence between the two types of peripheral cues used in Experiment 2 supports the argument that they compete for control of a common attentional resource. When two different peripheral cues, both of which normally attract attention to themselves automatically, are presented in close succession, mutual inhibition seems to work to direct attention to one or the other location, rather than divide it over several positions in space (Heinze et al., 1994; Juola, Bouwhuis, Cooper, \& Warner, 1991).

\section{REFERENCES}

Bonnel, A.-M., \& Bertucci, P. (1991, November). Brightness perception: Automatic detection versus controlled identification in concurrent tasks. Paper presented at the meeting of the Psychonomic Society, San Francisco.

Heinze, H.-J., Luck, S. J., Münte, T. F., Gös, A., Mangun, G.R., \& Hillyard, S. A. (1994). Attention to adjacent and separate positions in space: An electrophysiological analysis. Perception \& Psychophysics, 56, 42-52.

JONIDES, J. (1981). Voluntary versus automatic control over the mind's eye's movement. In J. B. Long \& A. D. Baddeley (Eds.), Attention and performance $I X$ (pp. 187-203). Hillsdale, NJ: Erlbaum.

JONIDES, J., \& YANTIS, S. (1988). Uniqueness of abrupt visual onset in capturing attention. Perception \& Psychophysics, 43, 346-354.

Juola, J. F., Bouwhuis, D. G., CoOPER, E. E., \& Warner, C. B. (1991). Control of attention around the fovea. Journal of Experimental Psychology: Human Perception \& Performance, 17, 125 141.

Koshino, H., Warner, C. B., \& Juola, J. F. (1992). Relative effectiveness of central, peripheral, and abrupt onset cues in visual attention. Quarterly Journal of Experimental Psychology, 45A, 609631.

MÜlleR, H. J., \& HuMPhreYs, G. W. (1991). Luminance-increment detection: Capacity-limited or not? Journal of Experimental Psychology: Human Perception \& Performance, 17, 107-124.

Müller, H. J., \& RabBITT, P. M. A. (1989). Reflexive and voluntary orienting of visual attention: Time course of activation and resistance to interruption. Journal of Experimental Psychology: Human Perception \& Performance, 15, 315-330.

POSNER, M. I. (1980). Orienting of attention. Quarterly Journal of Experimental Psychology, 32, 3-25.

THEEUWES, J. (1991). Exogenous and endogenous control of attention: The effect of visual onsets and offsets. Perception \& Psychophysics, 49, 83-90.

TODD, J. T., \& VAN GELDER, P. (1979). Implications of a transientsustained dichotomy for the measurement of human performance. Journal of Experimental Psychology: Human Perception \& Performance, 5, 625-638.

TSAL, Y. (1983). Movements of attention across the visual field. Journal of Experimental Psychology: Human Perception \& Performance, 9, 523-530.

WARner, C. B., Juola, J. F., \& Koshino, H. (1990). Voluntary allocation versus automatic capture of visual attention. Perception \& Psychophysics, 48, 243-251.

Yantis, S., \& Gibson, B. S. (1994). Object continuity in apparent motion and attention. Canadian Journal of Psychology, 48, 182204.

YANTIS, S., \& JoNiDES, J. (1984). Abrupt visual onset and selective at- 
tention: Evidence from visual search. Journal of Experimental Psychology: Human Perception \& Performance, 10, 601-621.

YANTIS, S., \& JoNidES, J. (1990). Abrupt visual onsets and selective attention: Voluntary vs. automatic allocation. Journal of Experimental Psychology: Human Perception \& Performance, 5,121-134.

\section{NOTE}

1. The costs plus benefits for each cue were determined by collapsing the same data set two different ways (i.e., across validity levels of the other cue). Thus, the data were not independent in the sense required by an ANOVA. This is not a serious problem for at least two rea- sons. First, each cue effect includes trials on which both cues were valid and both cues were invalid. These trials give a common lower and upper bound, respectively, for the cue effects, and they do not contribute to the overall ANOVA. Any effect therefore cannot be attributed to the common (nonindependent) elements, but rather to the unique effects of the validity of each cue when the other cue is valid or invalid. Second, all important interactions discussed in the costs-benefits analyses were also significant in the ANOVA of the raw RT data.

(Manuscript received July 8, 1993; revision accepted for publication October 10, 1994.) 